\title{
Efficient Syntheses of Diverse N-Heterocycles: The Molybdenum(VI)- Catalyzed Reductive Cyclization of Nitroarenes using Pinacol as a Deoxygenating Agent
}

\author{
Raghuram Gujjarappa ${ }^{a}$ \\ Nagaraju Vodnala ${ }^{a}$ \\ Arup K. Kabia \\ Dhananjaya Kaldhia \\ Mohan Kumara \\ Uwe Beifuss $^{\mathrm{b}}$ \\ Chandi C. Malakar*a (i) \\ ${ }^{a}$ Department of Chemistry, National Institute of Technology \\ Manipur, Langol, Imphal-795004, Manipur, India \\ cmalakar@nitmanipur.ac.in \\ b Institut für Chemie, Universität Hohenheim, Garbenstr. 30 \\ 70599 Stuttgart, Germany
}

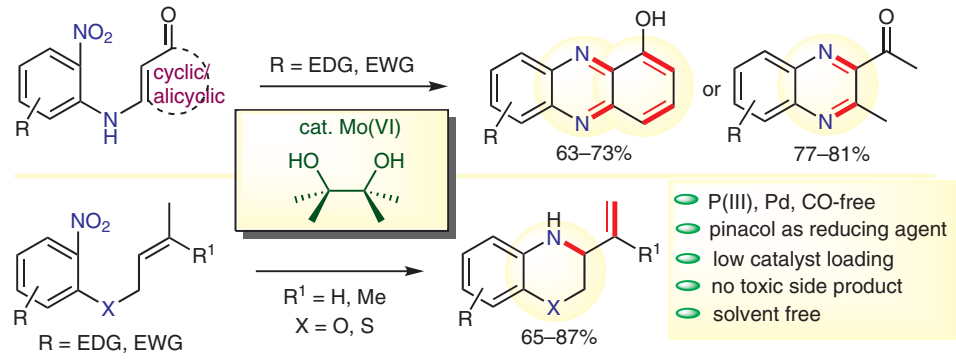

products are not easy to remove. Hence, the development of a more economical and environment-friendly process remains a challenge.

It is well-established that transition-metal complexes having multiple metal-oxygen bonds are efficient catalysts for deoxygenation reactions of organic compounds. ${ }^{10}$ In this regard, $\mathrm{MoO}_{2} \mathrm{Cl}_{2}(\mathrm{DMF})_{2}$ was found to be an efficient catalyst due to its ease of preparation in aqueous medium and low cost. ${ }^{11}$ Therefore, the redox properties of $\mathrm{MoO}_{2} \mathrm{Cl}_{2}(\mathrm{DMF})_{2}$ have been extensively studied for a broad spectrum of oxygen-transfer reactions, including our previous reports on reductive cyclization of nitroarenes. ${ }^{12}$ However, to accomplish these processes, a number of deoxygenating agents such as P(III)-based reagents, ${ }^{6}$ silanes, ${ }^{8 a}$ boranes, ${ }^{8 b}$ and molecular hydrogen ${ }^{8 c-d}$ have been used. Our attention was drawn towards a recent development from Sanz and coworkers, ${ }^{13}$ wherein they describe a novel method for the chemoselective reduction of sulfoxides and nitroaromatics using catalytic amounts of $\mathrm{Mo}(\mathrm{VI})$ in the presence of pinacol as a deoxygenating agent. Inspired by this report, we herein describe a method for the preparation of 1,4-benzoxazines, 1,4-benzothiazines, 1-hydroxyphenazines, and quinoxalines using $\mathrm{MoO}_{2} \mathrm{Cl}_{2}(\mathrm{DMF})_{2}$ as catalyst and pinacol as deoxygenating agent in 0 -xylene as solvent.

Six-membered $\mathrm{N}$-heterocycles such as 1,4-benzoxazines and 1,4-benzothiazines nucleus are well-known pharmacophoric scaffolds that have emerged as core structural units of a variety of antibacterial and antimicrobial agents. ${ }^{14} \mathrm{Sev}-$ eral approaches have been described for the syntheses of these scaffolds using $\omega$-nitroalkenes as substrates (Scheme 1). ${ }^{6}$ The reported protocols have been accomplished via the reductive cyclization of $\omega$-nitroalkenes using $\mathrm{P}(\mathrm{III})$-reagents and carbon monoxide as deoxygenating agents under both catalytic and non-catalytic conditions. The common drawbacks associated with these earlier approaches result from the use of excess of $\mathrm{P}(\mathrm{III})$-reagents and the formation of 
phosphine oxides in the reaction mixture. Moreover, when triethylphosphite was introduced as the deoxygenating agent, the corresponding $\mathrm{N}$-alkylated side products were observed in considerable amounts.
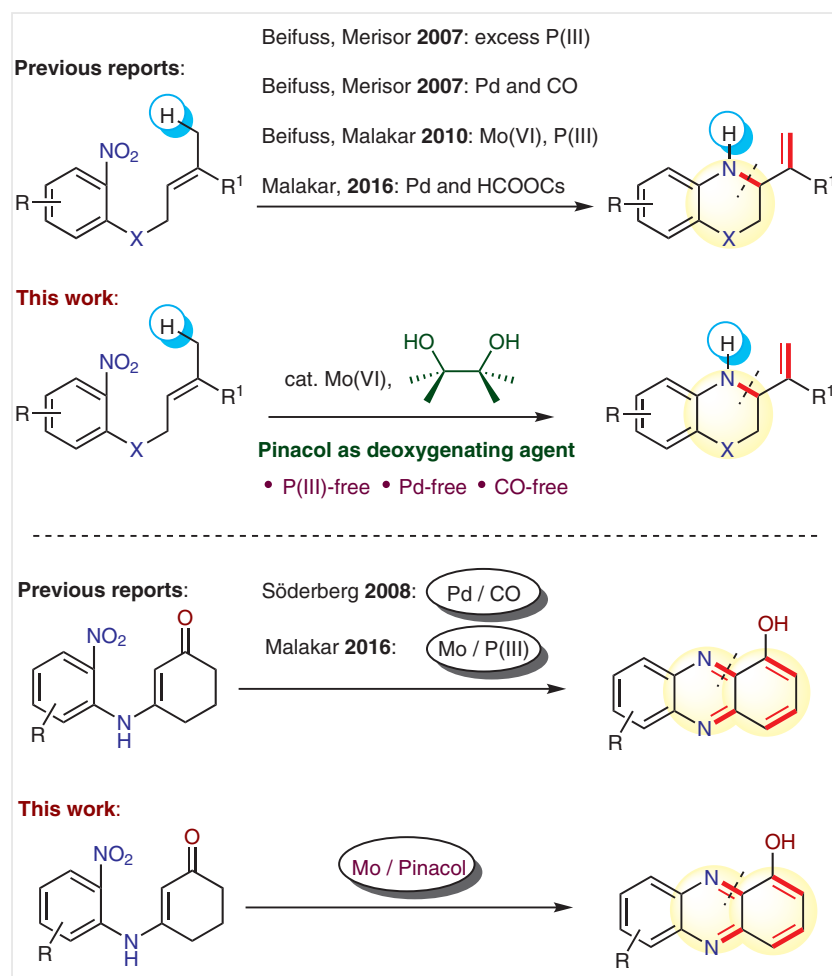

Scheme 1 Protocols for the synthesized scaffolds

The method reported herein has been extended towards the synthesis of 1-hydroxyphenazines and quinoxalines by the reductive cyclization of the appropriate $\beta$ - $(N-2$-nitroaryl)- $\alpha, \beta$-unsaturated ketones. Both 1-hydroxyphenazine and quinoxaline scaffolds have attracted considerable attention due to their unique biological activities such as antibacterial and DNA-cleaving properties. ${ }^{15}$ Additionally, these molecules have also been used as intermediates for the syntheses of more complex molecules. ${ }^{16}$

Starting materials $\mathbf{1} \mathbf{a}-\mathbf{i}$ and $\mathbf{3 a - f}$ were synthesized by using the previous reported methods. ${ }^{9 f, 12 \mathrm{~d}}$ In initial studies, 2-nitrophenyl ether 1a was used as a model substrate in the presence of $10 \mathrm{~mol} \% \mathrm{MoO}_{2} \mathrm{Cl}_{2}(\mathrm{DMF})_{2}$ as catalyst and pinacol as reducing agent in toluene at $110{ }^{\circ} \mathrm{C}$ for 15 hours in a sealed vial. Under these conditions, the desired product $3-$ isopropenyl-3,4-dihydro-2H-1,4-benzoxazine (2a) was obtained in $64 \%$ yield (Table 1 , entry 1 ). It could be demonstrated that, when alternative deoxygenating agents were employed with $10 \mathrm{~mol} \% \mathrm{Mo}(\mathrm{VI})$-catalyst, the expected product 2a was formed in lower yields (entries 3-6). We then investigated the optimum catalyst loading for the conversion of 1a into 2a. Similar yields of 2a were obtained with 5 mol\% and 2.5 mol\% Mo(VI)-catalyst (entries 7 and 8). However, when the catalyst loading was decreased beyond $2.5 \mathrm{~mol} \%$ the yield of the desired product $\mathbf{2 a}$ decreased (entries 9-11). A number of aromatic and nonaromatic solvents were then screened for the conversion of 1a into $\mathbf{2 a}$. Among the solvents examined, $\boldsymbol{o}$-xylene was most effective (entries 12-18). It was further observed that replacing $\mathrm{MoO}_{2} \mathrm{Cl}_{2}(\mathrm{DMF})_{2}$ with $\mathrm{MoO}_{2} \mathrm{Cl}_{2}$ as $\mathrm{Mo}(\mathrm{VI})$-source, resulted in the yield of 2a dropping dramatically (entry 19). When the reaction was studied under solvent-free conditions for a shorter reaction time (entries 20-22), it was found that the reaction of $1 \mathrm{a}$ in the presence of $2.5 \mathrm{~mol} \% \mathrm{MoO}_{2} \mathrm{Cl}_{2}(\mathrm{DMF})_{2}$ and 5.0 equiv of pinacol at $110{ }^{\circ} \mathrm{C}$ for 10 hours furnished the best yield (83\%) of the desired product $\mathbf{2 a}$ (entry 21 ). Therefore, these conditions were chosen as optimal conditions to establish the scope of the reaction.

Table 1 Screening of the Conditions for the $\mathrm{MoO}_{2} \mathrm{Cl}_{2}(\mathrm{DMF})_{2}$ Catalyzed Domino Reaction of $\mathbf{1 a}^{\mathrm{a}}$

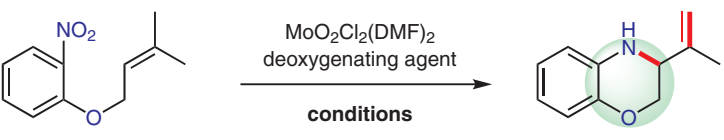


Table 1 (continued)

\begin{tabular}{|c|c|c|c|c|}
\hline Entry & Catalyst (mol\%) & Reagents (equiv) & Conditions & Yield 2a (\%) \\
\hline 9 & $\mathrm{MoO}_{2} \mathrm{Cl}_{2}(\mathrm{DMF})_{2}(1.5)$ & pinacol (2.0) & PhMe, $110^{\circ} \mathrm{C}, 15 \mathrm{~h}$ & 29 \\
\hline 10 & $\mathrm{MoO}_{2} \mathrm{Cl}_{2}(\mathrm{DMF})_{2}(0.5)$ & pinacol (2.0) & PhMe, $110^{\circ} \mathrm{C}, 15 \mathrm{~h}$ & 12 \\
\hline 11 & - & pinacol (2.0) & PhMe, $110^{\circ} \mathrm{C}, 20 \mathrm{~h}$ & $0^{c}$ \\
\hline 12 & $\mathrm{MoO}_{2} \mathrm{Cl}_{2}(\mathrm{DMF})_{2}(2.5)$ & pinacol (2.0) & $\mathrm{C}_{6} \mathrm{H}_{6}, 110^{\circ} \mathrm{C}, 15 \mathrm{~h}$ & 55 \\
\hline 13 & $\mathrm{MoO}_{2} \mathrm{Cl}_{2}(\mathrm{DMF})_{2}(2.5)$ & pinacol (2.0) & o-xylene, $130^{\circ} \mathrm{C}, 15 \mathrm{~h}$ & 73 \\
\hline 14 & $\mathrm{MoO}_{2} \mathrm{Cl}_{2}(\mathrm{DMF})_{2}(2.5)$ & - & PhMe, $110^{\circ} \mathrm{C}, 16 \mathrm{~h}$ & $9^{c}$ \\
\hline 15 & $\mathrm{MoO}_{2} \mathrm{Cl}_{2}(\mathrm{DMF})_{2}(2.5)$ & pinacol (2.0) & $\mathrm{MeCN}, 110^{\circ} \mathrm{C}, 15 \mathrm{~h}$ & 41 \\
\hline 16 & $\mathrm{MoO}_{2} \mathrm{Cl}_{2}(\mathrm{DMF})_{2}(2.5)$ & pinacol (2.0) & 1,4-dioxane, $140^{\circ} \mathrm{C}, 15 \mathrm{~h}$ & 68 \\
\hline 17 & $\mathrm{MoO}_{2} \mathrm{Cl}_{2}(\mathrm{DMF})_{2}(2.5)$ & pinacol (2.0) & DCE, $110^{\circ} \mathrm{C}, 15 \mathrm{~h}$ & 17 \\
\hline 18 & $\mathrm{MoO}_{2} \mathrm{Cl}_{2}(\mathrm{DMF})_{2}(2.5)$ & pinacol (2.0) & DME, $110^{\circ} \mathrm{C}, 15 \mathrm{~h}$ & 45 \\
\hline 19 & $\mathrm{MoO}_{2} \mathrm{Cl}_{2}(2.5)$ & pinacol (2.0) & PhMe, $110^{\circ} \mathrm{C}, 15 \mathrm{~h}$ & 45 \\
\hline 20 & $\mathrm{MoO}_{2} \mathrm{Cl}_{2}(\mathrm{DMF})_{2}(2.5)$ & pinacol (5.0) & $110^{\circ} \mathrm{C}, 15 \mathrm{~h}$ & $82^{d}$ \\
\hline 21 & $\mathrm{MoO}_{2} \mathrm{Cl}_{2}(\mathrm{DMF})_{2}(2.5)$ & pinacol (5.0) & $110^{\circ} \mathrm{C}, 10 \mathrm{~h}$ & $83^{d}$ \\
\hline 22 & $\mathrm{MoO}_{2} \mathrm{Cl}_{2}(\mathrm{DMF})_{2}(2.5)$ & pinacol (5.0) & $110^{\circ} \mathrm{C}, 7 \mathrm{~h}$ & $65^{d}$ \\
\hline
\end{tabular}

a Unless otherwise noted, all reactions were performed using $\mathbf{1 a}(1.0 \mathrm{mmol})$ in solvent $(2 \mathrm{~mL})$ in a sealed vial.

${ }^{b}$ Isolated yield.

' Unreacted starting material 1 a was recovered.

d Reactions were performed in neat condition.

When the optimized conditions were employed on a variety of $\omega$-nitroalkenes $\mathbf{1 a - i}$ (Scheme 2), it was found that both 2-nitroaryl ethers 1a-f and 2-nitroaryl thioethers $\mathbf{1 g -}$ i containing electron-withdrawing groups such as bromo, fluoro, and trifluoromethyl and the electron-donating groups such as methoxy and methyl on the arene-moiety were well tolerated, furnishing the desired 1,4-benzoxazines $\mathbf{2 a - f}$ and 1,4-benzothiazines $\mathbf{2 g}-\mathbf{i}$ in high yields ranging from 65 to $87 \%$. It is noteworthy that slightly decreased yields of $\mathbf{2}$ were obtained with the substrates having electron-withdrawing groups on the aromatic ring compared with nonsubstituted or electron-rich arenes. The reaction also proceeded successfully when the alkene was functionalized with a methyl group. Moreover, the feasibility of this protocol has been demonstrated for the gramscale synthesis of $\mathbf{2 g}$. Thus, $5.82 \mathrm{mmol}(1.3 \mathrm{~g})$ of 2-nitroaryl thioether $\mathbf{1 g}$ reacted under the optimized conditions to afford the corresponding cyclized product $2 \mathrm{~g}$ in $67 \%$ yield (3.9 $\mathrm{mmol} 746 \mathrm{mg}$ ).

Based on previous reports, ${ }^{13}$ a mechanistic proposal is shown in Scheme 3. In the first step, formation of the $\mathrm{MoO}$ (pinacolate) $\mathrm{Cl}_{2}(\mathrm{DMF})_{2}$ complex $\mathrm{C}$ can be realized by the reaction between $\mathrm{Mo}(\mathrm{VI})$-complex $\mathbf{A}$ and pinacol $\mathbf{B}$ on loss of a water molecule. Oxidative cleavage of the pinacolate ligands in complex $\mathbf{C}$ then delivers oxomolybdenum(IV) species $\mathbf{D}$, having a weakly coordinated acetone molecule that could be replaced by the oxygen-atom from $\omega$-nitroalkenes 1 to form the unstable Mo(IV)-species E. Next, cleavage of nitroso-aromatic $\mathbf{F}$ from the $\mathbf{M o}(\mathrm{IV})$-species $\mathbf{E}$ regenerates

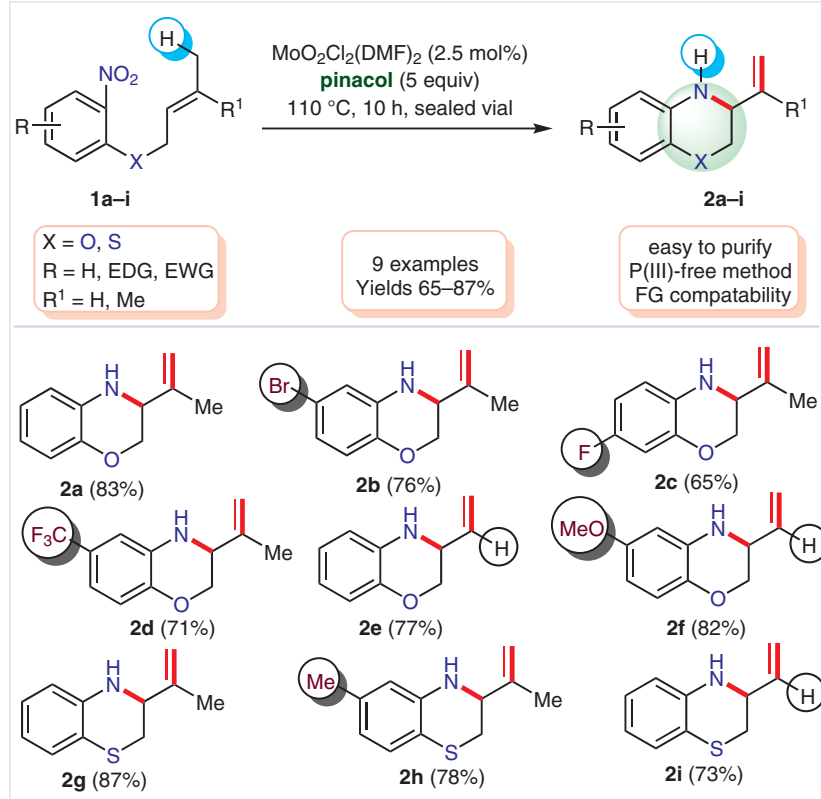

Scheme 2 Scope of the developed $\mathrm{MoO}_{2} \mathrm{Cl}_{2}(\mathrm{DMF})_{2}$-catalyzed domino approach for the synthesis of 1,4-benzoxazines $\mathbf{2 a -} \mathbf{f}$ and 1,4-benzothiazines $\mathbf{2 g}$-i. All reactions were performed using $1.0 \mathrm{mmol} \mathbf{1 a - i}$ under solvent-free conditions in sealed vial. Isolated yields are given.

the active $\mathrm{Mo}(\mathrm{VI})$-catalyst $\mathbf{A}$. The derived intermediate nitroso-aromatic $\mathbf{F}$ may undergo the nitroso-ene reaction to obtain the N-hydroxy compounds $\mathbf{G}$ which, on further deoxygenation, would release the final product 2 . 


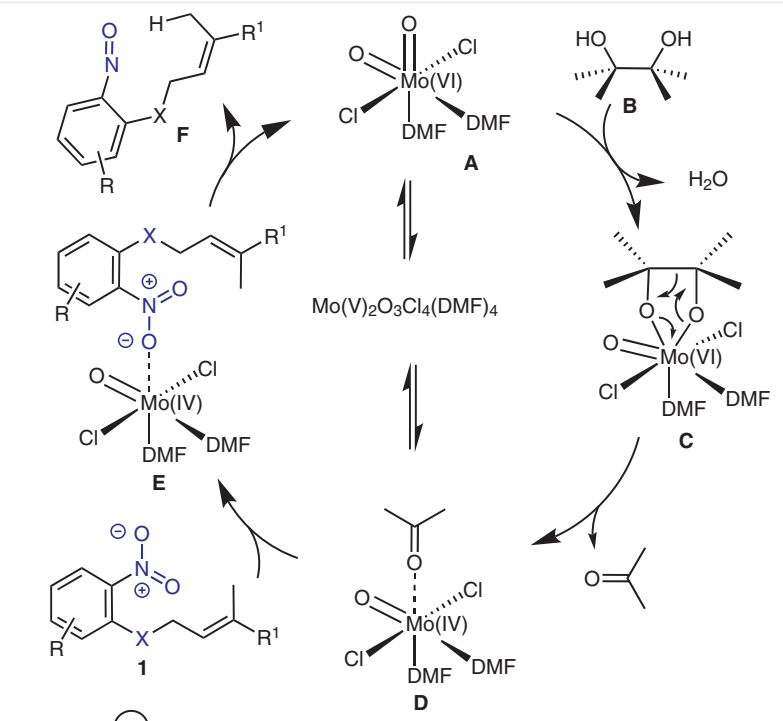

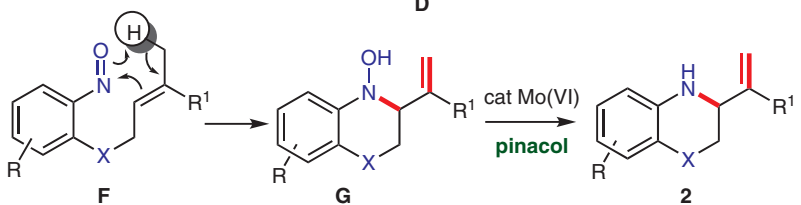

Scheme 3 Plausible mechanism for the Mo(VI)-catalyzed reductive cyclization using pinacol as a deoxygenating agent

To demonstrate further applications of this protocol, it was found that cyclic $\beta$-( $N$-2-nitroaryl)- $\alpha, \beta$-unsaturated ketones 3a-d can be employed under the optimized conditions to obtain 1-hydroxyphenazines 4a-d (Scheme 4). Cyclic $\beta$-( $N$-2-nitroaryl $)-\alpha, \beta$-unsaturated ketones having chloro, methoxy and methyl residues on the aromatic rings were well tolerated under the reaction conditions, delivering the desired 1-hydroxyphenazines $\mathbf{4 a - d}$ in yields ranging from 63 to 73\%. Furthermore, the protocol is not restricted to the synthesis of 1-hydroxyphenazines, but can be extended to the synthesis of quinoxaline derivatives. In this regard, acyclic $\beta$-( $N$-2-nitroaryl)- $\alpha, \beta$-unsaturated ketones reacted under the optimized reaction conditions to obtain quinoxalines $\mathbf{4 e - f}$ in yields ranging from 77 to $81 \%$ (Scheme 4). It is important to mention that only a limited number of methods are available for the synthesis of 1-hydroxyphenazines ${ }^{7 f, 17}$ including our previous report (Scheme 1), ${ }^{12 \mathrm{~d}}$ in which the reaction was carried out using catalytic $\mathrm{Mo}(\mathrm{VI})$ and $\mathrm{Ph}_{3} \mathrm{P}$ as deoxygenating agent. On the other hand, Söderberg and co-workers have described an approach towards the synthesis of the same scaffold using the Pd-catalyzed reductive cyclization of acyclic $\beta$-( $N$-2-nitroaryl)- $\alpha, \beta$-unsaturated ketones in the presence of $\mathrm{CO}$ as deoxygenating agent (Scheme 1). ${ }^{7 \mathrm{f}}$ Considering the advantages of the present method including low loading of inexpensive catalyst and the use of pinacol as deoxygenation reagent, this new protocol should find extensive application.

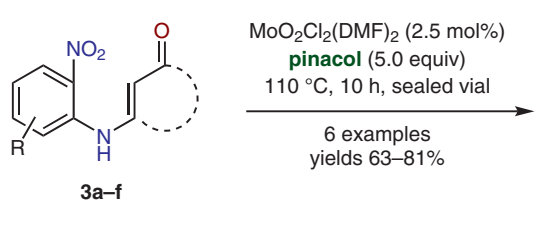<smiles>[R]c1cccc2nc(C(C)=O)c(C)nc12</smiles>

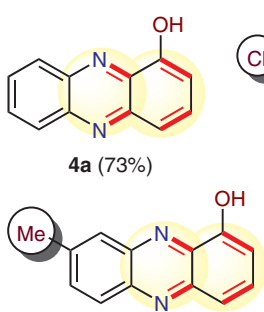

4d $(71 \%)$

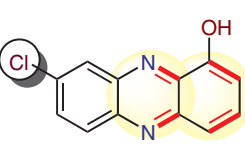

4b $(69 \%)$<smiles>CC(=O)c1nc2ccccc2nc1C</smiles>

4 e $(77 \%)$

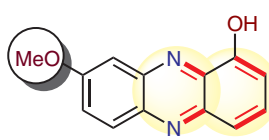

4c $(63 \%)$

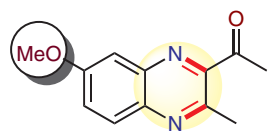

$4 f(81 \%)$
Scheme 4 Synthesis of 1-hydroxyphenazines and quinoxalines using $\mathrm{MoO}_{2} \mathrm{Cl}_{2}(\mathrm{DMF})_{2}$-catalyzed domino approach in the presence of pinacol as reducing agents. All reactions were performed using $\mathbf{3 a}-\mathbf{f}$ (1.0 $\mathrm{mmol}$ ) under solvent-free conditions in sealed vial. Isolated yields are given.

To summarize, we have demonstrated a domino reductive cyclization approach towards the synthesis of a broad spectrum of $\mathrm{N}$-heterocycles in the presence of low loadings of $\mathrm{Mo}(\mathrm{VI})$ complex as catalyst and pinacol as a readily available and inexpensive deoxygenating agent. The reactions are executed under aerobic and solvent-free conditions to furnish high isolated yields of the desired compounds with the formation of acetone and water as side products allowing for easy purification.

All starting materials were purchased from commercial suppliers (Sigma-Aldrich, Alfa-Aesar, SD fine chemicals, Merck, HI Media) and were used without further purification unless otherwise indicated. All reactions were carried out in oven-dried glassware with magnetic stirring in a sealed vial. Solvents used in extraction and purification were distilled prior to use. Thin-layer chromatography (TLC) was performed on TLC plates purchased from Merck. Compounds were visualized with UV light $(\lambda=254 \mathrm{~nm})$ and/or by immersion in $\mathrm{KMnO}_{4}$ solution followed by heating. Products were purified by flash column chromatography on silica gel, 230-400 mesh. IR spectra were measured with a Perkin-Elmer Spectrum One FT-IR spectrometer. ${ }^{1} \mathrm{H}\left({ }^{13} \mathrm{C}\right)$ NMR spectra were recorded at 300 (75.4) MHz with a Brucker spectrometer using $\mathrm{CDCl}_{3}$ as a solvent. Chemical shifts were referenced to residual solvent signals at $\delta_{\mathrm{H} / \mathrm{C}}=7.26 / 77.28 \mathrm{ppm}\left(\mathrm{CDCl}_{3}\right)$ relative to TMS as internal standards. Coupling constants $J[\mathrm{~Hz}]$ were directly taken from the spectra and are not averaged. Splitting patterns are designated as $\mathrm{s}$ ( singlet), $\mathrm{d}$ (doublet), $\mathrm{t}$ (triplet), q (quartet), $\mathrm{m}$ (multiplet) and br (broad). 


\section{Synthesis of Compounds 2a-i and 4a-f; General Procedure}

A $10 \mathrm{~mL}$ vial was charged with a mixture of $\mathbf{1 a}-\mathbf{i}$ or $\mathbf{3 a}-\mathbf{f}(1.0 \mathrm{mmol})$, $\mathrm{MoO}_{2} \mathrm{Cl}_{2}(\mathrm{dmf})_{2}(0.025 \mathrm{mmol})$ and pinacol $(5.0 \mathrm{mmol})$. The vial was then sealed and heated to $110{ }^{\circ} \mathrm{C}$ for $10 \mathrm{~h}$. After completion of the reaction (progress was monitored by TLC; $\mathrm{SiO}_{2}$, hexane/EtOAc $=20: 1$ for $\mathbf{2 a}-\mathbf{i}$ and hexane/EtOAC $=4: 1$ for $\mathbf{4 a - f}$ ), the mixture was diluted with hot EtOAc $(15 \mathrm{~mL})$ and water $(25 \mathrm{~mL})$ and extracted with EtOAc $(3 \times 10 \mathrm{~mL})$. The combined organic layers were washed with brine $(3 \times 10 \mathrm{~mL})$ and dried over anhydrous $\mathrm{Na}_{2} \mathrm{SO}_{4}$. After filtration, solvent was removed under reduced pressure and the remaining residue was purified by column chromatography over silica gel using hexane/EtO$A c=20: 1$ for $\mathbf{2} \mathbf{a}-\mathbf{i}$ and hexane/EtOAc $=4: 1$ for $\mathbf{4 a}-\mathbf{f}$ as an eluent to obtain the desired product $\mathbf{2 a - i}$ and $\mathbf{4 a - f}$ in high yields.

\section{3-Isopropenyl-3,4-dihydro-2H-benzo[1,4] oxazine (2a)}

Yield: 83\%; pale-yellow oil; $R_{f}=0.56\left(\mathrm{SiO}_{2}\right.$, hexane/EtOAc $\left.=20: 1\right)$. ${ }^{1} \mathrm{H}$ NMR $\left(300 \mathrm{MHz}, \mathrm{CDCl}_{3}\right): \delta=1.85\left(\mathrm{~s}, 3 \mathrm{H}, 3^{\prime}-\mathrm{H}_{3}\right), 3.97$ (overlapped, $1 \mathrm{H}, 3-\mathrm{H}), 3.95\left(\mathrm{dd},{ }^{3} \mathrm{~J}=7.6 \mathrm{~Hz},{ }^{2} \mathrm{~J}=16.8 \mathrm{~Hz}, 1 \mathrm{H}, 2-\mathrm{H}\right), 4.33\left(\mathrm{dd},{ }^{3} \mathrm{~J}=\right.$ $8.2 \mathrm{~Hz},{ }^{2} J=16.4 \mathrm{~Hz}, 1 \mathrm{H}, 2-\mathrm{H}$ ), 5.06 (brs, $1 \mathrm{H}, 2^{\prime}$-H), 5.17 (brs, $1 \mathrm{H}, 2^{\prime}$ H), 6.71, 6.73, 6.81, 6.84 (overlapped, 4 H, 5-H, 6-H, 7-H, 8-H).

${ }^{13} \mathrm{C}$ NMR $\left(75 \mathrm{MHz}, \mathrm{CDCl}_{3}\right): \delta=19.83\left(\mathrm{C}-3{ }^{\prime}\right), 55.38(\mathrm{C}-3), 68.75(\mathrm{C}-2)$, 113.61 (C-2'), 115.89 (C-5), 116.82 (C-8), 119.48 (C-7), 121.71 (C-6), 133.43 (C-10), $142.82\left(\mathrm{C}-1^{\prime}\right), 143.95$ (C-9).

\section{6-Bromo-3-(prop-1-en-2-yl)-3,4-dihydro-2H-benzo[b][1,4]ox- azine (2b) $)^{9 f}$}

Yield: 76\%; pale-yellow oil; $R_{f}=0.56\left(\mathrm{SiO}_{2}\right.$, hexane/EtOAc $\left.=20: 1\right)$; LCMS purity $99.3 \%$.

${ }^{1} \mathrm{H}$ NMR ( $\left.300 \mathrm{MHz}, \mathrm{CDCl}_{3}\right): \delta=1.80\left(\mathrm{~s}, 3 \mathrm{H}, 3^{\prime}-\mathrm{H}_{3}\right), 3.89$ (overlapped, $2 \mathrm{H}, 2-\mathrm{H}), 4.22(\mathrm{~m}, 1 \mathrm{H}, 3-\mathrm{H}), 5.01\left(\mathrm{~s}, 1 \mathrm{H}, 2^{\prime}-\mathrm{H}\right), 5.1$ (s, $\left.1 \mathrm{H}, 2^{\prime}-\mathrm{H}\right), 6.64$ $\left(\mathrm{d},{ }^{3} \mathrm{~J}=8.1 \mathrm{~Hz}, 1 \mathrm{H}, 8-\mathrm{H}\right), 6.71-6.74(\mathrm{~m}, 2 \mathrm{H}, 5-\mathrm{H}$ and $7-\mathrm{H})$.

\section{7-Fluoro-3-(prop-1-en-2-yl)-3,4-dihydro-2H-benzo[b][1,4]ox- azine (2c) $)^{9 \mathrm{f}}$}

Yield: 65\%; pale-yellow oil; $R_{f}=0.57\left(\mathrm{SiO}_{2}\right.$, hexane/EtOAc $\left.=20: 1\right)$.

${ }^{1} \mathrm{H}$ NMR $\left(300 \mathrm{MHz}, \mathrm{CDCl}_{3}\right): \delta=1.81\left(\mathrm{~s}, 3 \mathrm{H}, 3^{\prime}-\mathrm{H}_{3}\right), 3.83-3.93(\mathrm{~m}, 2 \mathrm{H}$, 2-H), 4.22-4.26 (m, $1 \mathrm{H}, 3-\mathrm{H}), 5.01\left(\mathrm{~s}, 1 \mathrm{H}, 2^{\prime}-\mathrm{H}\right), 5.10\left(\mathrm{~s}, 1 \mathrm{H}, 2^{\prime}-\mathrm{H}\right)$, 6.47-6.56 (m, 3 H, 5-H, 6-H and 8-H).

\section{3-(Prop-1-en-2-yl)-6-(trifluoromethyl)-3,4-dihydro-2H-ben- zo[b][1,4] oxazine (2d) ${ }^{9 f}$}

Yield: 71\%; pale-yellow oil; $R_{f}=0.58\left(\mathrm{SiO}_{2}\right.$, hexane/EtOAc $\left.=20: 1\right)$; LCMS purity $99.7 \%$.

${ }^{1} \mathrm{H}$ NMR $\left(300 \mathrm{MHz}, \mathrm{CDCl}_{3}\right): \delta=1.82\left(\mathrm{~s}, 3 \mathrm{H}, 3^{\prime}-\mathrm{H}_{3}\right), 3.89-3.96(\mathrm{~m}, 2 \mathrm{H}$, 2-H), 4.27-4.29 (m, $1 \mathrm{H}, 3-\mathrm{H}), 5.03$ (s, $\left.1 \mathrm{H}, 2^{\prime}-\mathrm{H}\right), 5.09$ (s, $\left.1 \mathrm{H}, 2^{\prime}-\mathrm{H}\right)$, 6.82-6.90 (m, 3 H, 5-H, 7-H and 8-H).

\section{3-Vinyl-3,4-dihydro-2H-benzo[1,4]oxazine $(2 \mathrm{e})^{9 \mathrm{f}}$}

Yield: 77\%; pale-yellow oil; $R_{f}=0.45\left(\mathrm{SiO}_{2}\right.$, hexane/EtOAc $\left.=20: 1\right)$.

${ }^{1} \mathrm{H}$ NMR $\left(300 \mathrm{MHz}, \mathrm{CDCl}_{3}\right.$ ): $\delta=3.8$ (overlapped, $1 \mathrm{H}, 2-\mathrm{H}$ ), 4.07 (overlapped, $1 \mathrm{H}, 3-\mathrm{H}), 4.28\left(\mathrm{dd},{ }^{3} \mathrm{~J}=9.8 \mathrm{~Hz}, 1 \mathrm{H}, 2-\mathrm{H}\right), 5.31$ (brd, ${ }^{3} \mathrm{~J}=$ $10.3 \mathrm{~Hz}, 1 \mathrm{H}, 2^{\prime}-\mathrm{H}$ ), 5.45 (brd, $\left.{ }^{2} J=17.2 \mathrm{~Hz}, 1 \mathrm{H}, 2^{\prime}-\mathrm{H}\right), 5.91$ (m, $1 \mathrm{H}, 1^{\prime}-$ $\mathrm{H}), 6.67\left(\mathrm{dd},{ }^{3} \mathrm{~J}=7.7 \mathrm{~Hz}, 1 \mathrm{H}, 8-\mathrm{H}\right), 6.73\left(\mathrm{dd},{ }^{3} \mathrm{~J}=7.7 \mathrm{~Hz},{ }^{4} \mathrm{~J}=1.5 \mathrm{~Hz}, 1 \mathrm{H}\right.$, 7-H), 6.76-6.85 (overlapped, $2 \mathrm{H}, 5-\mathrm{H}$ and 6-H).

${ }^{13} \mathrm{C}$ NMR (75 MHz, $\mathrm{CDCl}_{3}$ ): $\delta=52.22(\mathrm{C}-3), 69.14(\mathrm{C}-2), 115.75(\mathrm{C}-8)$, 116.86 (C-5), 118.23 (C-2'), 119.24 (C-7), 121.69 (C-6), 133.18 (C-10), $135.63\left(\mathrm{C}-1^{\prime}\right), 143.79(\mathrm{C}-9)$.
6-Methoxy-3-vinyl-3,4-dihydro-2H-benzo[b][1,4] oxazine (2f $)^{\text {9f }}$

Yield: $82 \%$; pale-yellow oil; $R_{f}=0.46\left(\mathrm{SiO}_{2}\right.$, hexane/EtOAc $\left.=20: 1\right)$; LCMS purity $98.7 \%$.

${ }^{1} \mathrm{H}$ NMR $\left(300 \mathrm{MHz}, \mathrm{CDCl}_{3}\right): \delta=3.76(\mathrm{~s}, 3 \mathrm{H}, 11-\mathrm{H}), 3.82-3.87(\mathrm{~m}, 1 \mathrm{H}$, 2-H), 3.94-3.96 (m, $1 \mathrm{H}, 3-\mathrm{H}), 4.15-4.19(\mathrm{~m}, 1 \mathrm{H}, 2-\mathrm{H}), 5.25\left(\mathrm{~d},{ }^{3} \mathrm{~J}=\right.$ $\left.16.1 \mathrm{~Hz}, 1 \mathrm{H}, 2^{\prime}-\mathrm{H}\right), 5.37\left(\mathrm{~d},{ }^{2} \mathrm{~J}=17.2 \mathrm{~Hz}, 1 \mathrm{H}, 2^{\prime}-\mathrm{H}\right), 5.78-5.87(\mathrm{~m}, 1 \mathrm{H}$, $\left.1^{\prime}-\mathrm{H}\right), 6.20-6.23$ (m, $2 \mathrm{H}, 5-\mathrm{H}$ and 7-H), $6.70\left(\mathrm{~d},{ }^{3} \mathrm{~J}=8.2 \mathrm{~Hz}, 1 \mathrm{H}, 8 \mathrm{H}\right)$.

\section{3-Isopropenyl-3,4-dihydro-2H-benzo[1,4]thiazine (2g) $)^{9 f, 12 a}$}

Yield: 87\%; light-yellow oil; $R_{f}=0.56\left(\mathrm{SiO}_{2}\right.$, hexane/EtOAc $\left.=20: 1\right)$. ${ }^{1} \mathrm{H}$ NMR $\left(300 \mathrm{MHz}, \mathrm{CDCl}_{3}\right): \delta=1.85\left(\mathrm{~s}, 3 \mathrm{H}, 3^{\prime}-\mathrm{H}_{3}\right), 2.97\left(\mathrm{dd},{ }^{3} \mathrm{~J}=3.9 \mathrm{~Hz}\right.$, ${ }^{2} J=12.5 \mathrm{~Hz}, 1 \mathrm{H}, 2-\mathrm{H}$ ), 3.00 (dd, ${ }^{3} J=7.3 \mathrm{~Hz},{ }^{2} J=12.5 \mathrm{~Hz}, 1 \mathrm{H}, 2-\mathrm{H}$ ), 4.06 (brdd, ${ }^{3} \mathrm{~J}=3.9 \mathrm{~Hz},{ }^{3} \mathrm{~J}=7.2 \mathrm{~Hz}, 1 \mathrm{H}, 3-\mathrm{H}$ ), 5.05 (brs, $1 \mathrm{H}, 2^{\prime}-\mathrm{H}$ ), 5.14 (brs, $\left.1 \mathrm{H}, 2^{\prime}-\mathrm{H}\right), 6.52\left(\mathrm{dd},{ }^{3} \mathrm{~J}=8.0 \mathrm{~Hz},{ }^{2} \mathrm{~J}=1.3 \mathrm{~Hz}, 1 \mathrm{H}, 5-\mathrm{H}\right), 6.65\left(\mathrm{ddd},{ }^{3} \mathrm{~J}=\right.$ $\left.7.5 \mathrm{~Hz},{ }^{3} \mathrm{~J}=7.5 \mathrm{~Hz},{ }^{2} \mathrm{~J}=1.3 \mathrm{~Hz}, 1 \mathrm{H}, 7-\mathrm{H}\right), 6.91\left(\mathrm{ddt},{ }^{3} \mathrm{~J}=7.3 \mathrm{~Hz},{ }^{3} \mathrm{~J}=\right.$ $\left.8.0 \mathrm{~Hz},{ }^{2} \mathrm{~J}=1.6 \mathrm{~Hz}, 1 \mathrm{H}, 6-\mathrm{H}\right), 7.03\left(\mathrm{dd},{ }^{3} \mathrm{~J}=7.8 \mathrm{~Hz},{ }^{2} \mathrm{~J}=1.5 \mathrm{~Hz}, 1 \mathrm{H}, 8-\mathrm{H}\right)$. ${ }^{13} \mathrm{C}$ NMR $\left(75 \mathrm{MHz}, \mathrm{CDCl}_{3}\right): \delta=19.24\left(\mathrm{C}-3^{\prime}\right), 30.17$ (C-2), $57.18(\mathrm{C}-3)$, 112.93 (C-2'), 115.63 (C-5), 115.89 (C-9), 118.53 (C-7), 125.95 (C-6), 127.72 (C-8), 142.06 (C-10), $145.79\left(\mathrm{C}-1^{\prime}\right)$.

MS (EI, $70 \mathrm{eV}): m / z(\%)=191.1(100)\left[\mathrm{M}^{+}\right], 163.1(18), 150.1$ (46), 117.1 (21), $109.0(11), 65(5)$.

\section{3-Isopropenyl-6-methyl-3,4-dihydro-2H-benzo[1,4]thiazine} (2h) $)^{9 \mathrm{f}, 12 \mathrm{a}}$

Yield: 78\%; pale-yellow oil; $R_{f}=0.58\left(\mathrm{SiO}_{2}\right.$, hexane/EtOAc $\left.=20: 1\right)$. ${ }^{1} \mathrm{H} \mathrm{NMR}\left(300 \mathrm{MHz}, \mathrm{CDCl}_{3}\right): \delta=1.83\left(\mathrm{~s}, 3 \mathrm{H}, 3^{\prime}-\mathrm{H}_{3}\right), 2.23\left(\mathrm{~s}, 3 \mathrm{H}, 11-\mathrm{H}_{3}\right)$, 2.97-2.99 (overlapped, $2 \mathrm{H}, 2-\mathrm{H}_{2}$ ), 4.06 (brdd, ${ }^{3} \mathrm{~J}=3.9 \mathrm{~Hz},{ }^{3} \mathrm{~J}=7.2 \mathrm{~Hz}$, $1 \mathrm{H}, 3-\mathrm{H}), 5.04$ (brs, $\left.1 \mathrm{H}, 2^{\prime}-\mathrm{H}\right), 5.07$ (brs, $\left.1 \mathrm{H}, 2^{\prime}-\mathrm{H}\right), 6.37$ (s, $1 \mathrm{H}, 5-\mathrm{H}$ ), $6.48\left(\mathrm{ddd},{ }^{3} \mathrm{~J}=7.5 \mathrm{~Hz},{ }^{3} \mathrm{~J}=7.6 \mathrm{~Hz},{ }^{4} \mathrm{~J}=1.2 \mathrm{~Hz}, 1 \mathrm{H}, 7-\mathrm{H}\right), 6.03\left(\mathrm{dd},{ }^{3} \mathrm{~J}=\right.$ $\left.7.6 \mathrm{~Hz},{ }^{4} J=1.4 \mathrm{~Hz}, 1 \mathrm{H}, 8-\mathrm{H}\right)$.

${ }^{13} \mathrm{C}$ NMR (75 MHz, CDCl 3 ): $\delta=18.93\left(\mathrm{C}-3^{\prime}\right), 21.03(\mathrm{C}-11), 30.05(\mathrm{C}-2)$, 57.16 (C-3), 112.08 (C-2'), 112.53 (C-5), 115.84 (C-9), 119.14 (C-7), $127.43(\mathrm{C}-6), 135.57(\mathrm{C}-8), 141.75(\mathrm{C}-10), 145.83\left(\mathrm{C}-1^{\prime}\right)$

MS (EI, $70 \mathrm{eV}): m / z(\%)=205.1(100)\left[\mathrm{M}^{+}\right], 206.1(20), 190.1(24)$, 177.1 (16), 164.1 (40), 158.1 (9), 131.1 (2), 44 (2).

\section{3-Vinyl-3,4-dihydro-2H-benzo[ 1,4$]$ thiazine $(2 \mathrm{i})^{9 f, 12 \mathrm{a}}$}

Yield: 73\%; pale-yellow oil; $R_{f}=0.44\left(\mathrm{SiO}_{2}\right.$, hexane/EtOAc $\left.=20: 1\right)$.

${ }^{1} \mathrm{H}$ NMR $\left(300 \mathrm{MHz}, \mathrm{CDCl}_{3}\right.$ ): $\delta=2.97$ (overlapped, ${ }^{3} \mathrm{~J}=11.2 \mathrm{~Hz}, 2 \mathrm{H}, 2-$ H), 4.12 (overlapped, $1 \mathrm{H}, 3-\mathrm{H}$ ), 5.21 (d, ${ }^{3} \mathrm{~J}=10.5 \mathrm{~Hz}, 1 \mathrm{H}, 2^{\prime}-\mathrm{H}$ ), 5.34 (d, $\left.{ }^{2} J=17.1 \mathrm{~Hz}, 1 \mathrm{H}, 2^{\prime}-\mathrm{H}\right), 5.88\left(\mathrm{~m}, 1 \mathrm{H}, 1^{\prime}-\mathrm{H}\right), 6.49\left(\mathrm{~d},{ }^{3} \mathrm{~J}=8.1 \mathrm{~Hz}, 1 \mathrm{H}, 5-\right.$ $\mathrm{H}), 6.62\left(\mathrm{td},{ }^{3} \mathrm{~J}=6.3 \mathrm{~Hz},{ }^{4} \mathrm{~J}=1.3 \mathrm{~Hz}, 1 \mathrm{H}, 7-\mathrm{H}\right), 6.93\left(\mathrm{td},{ }^{3} \mathrm{~J}=11.7 \mathrm{~Hz},{ }^{4} \mathrm{~J}=\right.$ $1.2 \mathrm{~Hz}, 1 \mathrm{H}, 6-\mathrm{H}), 7.05$ (dd, $\left.{ }^{3} \mathrm{~J}=10.5 \mathrm{~Hz}, 1 \mathrm{H}, 8-\mathrm{H}\right)$.

${ }^{13} \mathrm{C}$ NMR (75.4 MHz, $\mathrm{CDCl}_{3}$ ): $\delta=30.93(\mathrm{C}-2), 54.36(\mathrm{C}-3), 115.71(\mathrm{C}-5$, C-2' overlapped), 117.21 (C-9), 118.63 (C-7), 125.91 (C-6), 127.76 (C8), $138.94\left(\mathrm{C}-1^{\prime}\right), 141.83$ (C-10).

MS (EI, $70 \mathrm{eV}): m / z(\%)=177(100)\left[\mathrm{M}^{+}\right], 162.1(82), 149.1$ (66), 144.1 (31), 130.10 (17),117.1 (9), 109 (4).

\section{1-Hydroxyphenazine $(4 a)^{12 d}$}

Yield: 73\%; yellow solid; $R_{f}=0.30\left(\mathrm{SiO}_{2}\right.$, hexane/EtOAc $\left.=4: 1\right) ; \mathrm{mp}$ $157-159{ }^{\circ} \mathrm{C}$ (Lit. $\left.{ }^{7 f} 153-155^{\circ} \mathrm{C}\right)$.

${ }^{1} \mathrm{H}$ NMR $\left(300 \mathrm{MHz}, \mathrm{CDCl}_{3}\right): \delta=7.77-7.88(\mathrm{~m}, 4 \mathrm{H}, 5-\mathrm{H}, 6-\mathrm{H}, 7-\mathrm{H}$ and $8-$ $\mathrm{H}), 8.21-8.29(\mathrm{~m}, 3 \mathrm{H}, 2-\mathrm{H}, 3-\mathrm{H}$ and $4-\mathrm{H})$.

${ }^{13} \mathrm{C}$ NMR (75 MHz, $\mathrm{CDCl}_{3}$ ): $\delta=109.11(\mathrm{C}-2), 120.21(\mathrm{C}-3), 129.46$ (C6), 129.989 (C-7), 130.75 (C-8), 131.03 (C-5), 132.09 (C-4), 134.51 (C1), 141.2 (C-11), 144.1 (C-13), 144.3 (C-14), 151.8 (C-12). 
MS (GC-MS): $m / z(\%)=197(12)[\mathrm{M}+1]^{+}, 196(100)\left[\mathrm{M}^{+}\right], 168(88), 140$ (8), $114(5), 102(5), 77(12)\left[\mathrm{C}_{6} \mathrm{H}_{5}\right]^{+}$.

\section{7-Chloro-1-hydroxyphenazine (4b) $)^{12 \mathrm{~d}}$}

Yield: $69 \%$; yellow solid; $R_{f}=0.29\left(\mathrm{SiO}_{2}\right.$, hexane/EtOAc $\left.=4: 1\right) ; \mathrm{mp}$ $181-183^{\circ} \mathrm{C}$

IR (ATR): 2159 (m; O-H), 1511 (m; alkane C-H), 1480 (m), 1400 (m), $1174(\mathrm{~s}), 1120(\mathrm{~m}), 1095$ (m), 830 (m; arom. C-H), $760 \mathrm{~cm}^{-1}(\mathrm{~s})$.

$\mathrm{UV} / \mathrm{Vis}\left(\mathrm{CH}_{3} \mathrm{CN}\right): \lambda_{\max }(\log \varepsilon)=207$ (3.51), 265 (3.76), $373 \mathrm{~nm}$ (2.69).

${ }^{1} \mathrm{H}$ NMR (300 MHz, $\mathrm{CDCl}_{3}$ ): $\delta=7.25$ (overlapped, $1 \mathrm{H}, 2-\mathrm{H}$ ), 7.76-7.80 ( $\mathrm{m}, 3 \mathrm{H}, 3-\mathrm{H}, 4-\mathrm{H}$ and 5-H), 8.1 (s, $1 \mathrm{H}, 6-\mathrm{H}$ ), 8.22 (overlapped, $2 \mathrm{H}, 8-$ $\mathrm{H}$ and $\mathrm{OH}$ ).

${ }^{13} \mathrm{C}$ NMR (75 MHz, $\mathrm{CDCl}_{3}$ ): $\delta=109.63(\mathrm{C}-2), 120.03$ (C-3), $127.54(\mathrm{C}-$ 6), 130.99 (C-8), 132.13 (C-5), 132.16 (C-4), 134.89 (C-1), 136.59 (C7), 141.01 (C-11), 142.57 (C-13), 143.76 (C-14), 151.57 (C-12).

MS (EI, $70 \mathrm{eV}): m / z(\%)=232(14)[\mathrm{M}+2]^{+}, 230(39)\left[\mathrm{M}^{+}\right], 202(20)$ $\left[\mathrm{C}_{11} \mathrm{H}_{7} \mathrm{ClN}_{2}\right]^{+}, 167(12)\left[\mathrm{C}_{11} \mathrm{H}_{7} \mathrm{~N}_{2}\right]^{+}, 149(9), 114(19), 97(9)$.

HRMS (EI, $\mathrm{M}^{+}$): $m / z$ calcd for $\mathrm{C}_{12} \mathrm{H}_{7} \mathrm{OClN}_{2}$ : 230.0247; found: 230.0207 .

\section{7-Methoxy-1-hydroxyphenazine (4c) ${ }^{12 d}$}

Yield: $63 \%$; yellow solid; $R_{f}=0.27\left(\mathrm{SiO}_{2}\right.$, hexane/EtOAc $\left.=4: 1\right) ; \mathrm{mp}$ $179-182{ }^{\circ} \mathrm{C}$

IR (ATR): 2922 (w; $\left.\mathrm{CH}_{3}\right), 1615$ (m), 1485 (s; alkane C-H), 1212 (s), $1024(\mathrm{~m}), 874(\mathrm{~m}), 808(\mathrm{~s}), 740 \mathrm{~cm}^{-1}(\mathrm{~m})$

$\mathrm{UV} / \mathrm{Vis}\left(\mathrm{CH}_{3} \mathrm{CN}\right): \lambda_{\max }(\log \varepsilon)=212$ (3.68), 265 (4.0), $377 \mathrm{~nm}$ (3.17).

${ }^{1} \mathrm{H}$ NMR $\left(300 \mathrm{MHz}, \mathrm{CDCl}_{3}\right): \delta=4.03\left(\mathrm{~s}, 3 \mathrm{H}, 15-\mathrm{H}_{3}\right), 7.17\left(\mathrm{dd},{ }^{3} \mathrm{~J}(5-\mathrm{H}, 6-\right.$ $\left.\mathrm{H})=5.1 \mathrm{~Hz},{ }^{4} \mathrm{~J}(6-\mathrm{H}, 8-\mathrm{H})=1.5 \mathrm{~Hz}, 1 \mathrm{H}, 6-\mathrm{H}\right), 7.49$ (overlapped, $2 \mathrm{H}, 2-$ $\mathrm{H}$ and 3-H), 7.68-7.77 ( $\mathrm{m}, 2 \mathrm{H}, 4-\mathrm{H}$ and 5-H), 8.10 (overlapped, $2 \mathrm{H}, 8$ $\mathrm{H}$ and $\mathrm{OH}$ ).

${ }^{13} \mathrm{C}$ NMR (75 MHz, $\mathrm{CDCl}_{3}$ ): $\delta=55.98(\mathrm{C}-15), 104.58(\mathrm{C}-8), 108.02(\mathrm{C}-$ 2), 119.09 (C-3), 126.36 (C-6), 130.0 (C-5), 130.18 (C-4), 131.03 (C-1), 132.91 (C-11), 138.39 (C-13), $146.3(\mathrm{C}-14), 151.92(\mathrm{C}-12), 161.6(\mathrm{C}-7)$. MS (EI, $70 \mathrm{eV}): m / z(\%)=227(14)[\mathrm{M}+1]^{+}, 226(100)\left[\mathrm{M}^{+}\right], 198(28)$ $[\mathrm{M}-\mathrm{CO}]^{+}, 183(19)\left[\mathrm{C}_{11} \mathrm{H}_{7} \mathrm{~N}_{2} \mathrm{O}\right]^{+}, 155(22), 114(18), 72(20), 59(28)$.

HRMS (EI, M+): $m / z$ calcd for $\mathrm{C}_{13} \mathrm{H}_{10} \mathrm{~N}_{2} \mathrm{O}_{2}$ : 226.0743; found: 226.0736 .

\section{7-Methyl-1-hydroxyphenazine (4d) ${ }^{12 d}$}

Yield: $71 \%$; yellow solid; $R_{f}=0.28\left(\mathrm{SiO}_{2}\right.$, hexane/EtOAc $\left.=4: 1\right) ; \mathrm{mp}=$ $163-166{ }^{\circ} \mathrm{C}$

${ }^{1} \mathrm{H}$ NMR $\left(300 \mathrm{MHz}, \mathrm{CDCl}_{3}\right): \delta=2.67\left(\mathrm{~s}, 3 \mathrm{H}, 15-\mathrm{H}_{3}\right), 7.22$ (overlapped, $1 \mathrm{H}, 2-\mathrm{H}), 7.66-7.76(\mathrm{~m}, 3 \mathrm{H}, 3-\mathrm{H}, 4-\mathrm{H}$ and $5-\mathrm{H}), 7.99\left(\mathrm{~d},{ }^{3} \mathrm{~J}(5-\mathrm{H}, 6-\mathrm{H})=\right.$ $9.3 \mathrm{~Hz}, 1 \mathrm{H}, 6-\mathrm{H}$ ), 8.15 (overlapped, $2 \mathrm{H}, 8-\mathrm{H}$ and $\mathrm{OH}$ ).

\section{1-(3-Methylquinoxalin-2-yl)ethanone (4e) ${ }^{12 \mathrm{~d}}$}

Yield: 77\%; colorless solid; $R_{f}=0.46\left(\mathrm{SiO}_{2}\right.$, hexane/EtOAc $\left.=4: 1\right) ; \mathrm{mp}$ $80-82{ }^{\circ} \mathrm{C}$ ( Lit. $^{17 \mathrm{a}} 79-81^{\circ} \mathrm{C}$ ).

${ }^{1} \mathrm{H}$ NMR $\left(300 \mathrm{MHz}, \mathrm{CDCl}_{3}\right): \delta=2.83\left(\mathrm{~s}, 3 \mathrm{H}, 13-\mathrm{H}_{3}\right), 2.96(\mathrm{~s}, 3 \mathrm{H}, 12-$ $\left.\mathrm{H}_{3}\right), 7.72-7.85(\mathrm{~m}, 2 \mathrm{H}, 6-\mathrm{H}$ and $7-\mathrm{H}), 8.02\left(\mathrm{~d},{ }^{3} \mathrm{~J}(5-\mathrm{H}, 6-\mathrm{H})=8.1 \mathrm{~Hz}\right.$, $1 \mathrm{H}, 5-\mathrm{H}), 8.09\left(\mathrm{dd},{ }^{3} \mathrm{~J}(7-\mathrm{H}, 8-\mathrm{H})=8.1 \mathrm{~Hz},{ }^{4} J(6-\mathrm{H}, 8-\mathrm{H})=1.2 \mathrm{~Hz}, 1 \mathrm{H}, 8-\right.$ $\mathrm{H})$.

${ }^{13} \mathrm{C}$ NMR (75 MHz, CDCl $)$ ): $\delta=24.69$ (C-13), 28.05 (C-12), 128.68 (C5), 129.82 (C-6), 130.09 (C-7), 132.24 (C-8), 140.03 (C-10), 143.1 (C9), 147.8 (C-3), 153.72 (C-2), 201.9 (C-11).
1-(7-Methoxy-3-methylquinoxalin-2-yl)ethanone (4f) $)^{12 \mathrm{~d}}$

Yield: $81 \%$; colorless solid; $R_{f}=0.43\left(\mathrm{SiO}_{2}\right.$, hexane/EtOAc $\left.=4: 1\right) ; \mathrm{mp}$ $87-90{ }^{\circ} \mathrm{C}$.

IR (ATR): 3140 (w; $\mathrm{CH}_{3}$ ), 1693 (s; C=0), 1616 (m; alkane C-H), 1491 (m), $1411(\mathrm{~m}), 1362(\mathrm{~m}$; alkane C-H), 1316 (m; alkane C-H), $1216(\mathrm{~s})$, $1126(\mathrm{~m}), 1059$ (m), 1027 (m), 937 (m; arom. C-H), $845 \mathrm{~cm}^{-1}$ (s; arom. C-H).

$\mathrm{UV} / \mathrm{Vis}\left(\mathrm{CH}_{3} \mathrm{CN}\right): \lambda_{\max }(\log \varepsilon)=219$ (3.64), 251 (3.75), $357 \mathrm{~nm}(2.82)$.

${ }^{1} \mathrm{H}$ NMR $\left(300 \mathrm{MHz}, \mathrm{CDCl}_{3}\right): \delta=2.83\left(\mathrm{~s}, 3 \mathrm{H}, 13-\mathrm{H}_{3}\right), 2.92(\mathrm{~s}, 3 \mathrm{H}, 12-$ $\left.\mathrm{H}_{3}\right), 3.99\left(\mathrm{~s}, 3 \mathrm{H}, 14-\mathrm{H}_{3}\right), 7.36\left(\mathrm{~d},{ }^{4} J(6-\mathrm{H}, 8-\mathrm{H})=2.7 \mathrm{~Hz}, 1 \mathrm{H}, 8-\mathrm{H}\right), 7.48$ $\left(\mathrm{dd},{ }^{3} J(5-\mathrm{H}, 6-\mathrm{H})=6.6 \mathrm{~Hz},{ }^{4} J(6-\mathrm{H}, 8-\mathrm{H})=2.4 \mathrm{~Hz}, 1 \mathrm{H}, 6-\mathrm{H}\right), 7.92(\mathrm{~d}$, $\left.{ }^{3} J(5-\mathrm{H}, 6-\mathrm{H})=9.3 \mathrm{~Hz}, 1 \mathrm{H}, 5-\mathrm{H}\right)$.

${ }^{13} \mathrm{C}$ NMR (75 MHz, $\mathrm{CDCl}_{3}$ ): $\delta=24.01(\mathrm{C}-13), 27.85$ (C-12), 55.85 (C14), 106.67 (C-8), 125.51 (C-5), 129.31 (C-6), 138.99 (C-10), 141.36 (C-9), 147.06 (C-3), 150.37 (C-2), 160.47 (C-7), 201.55 (C-11).

MS (EI, $70 \mathrm{eV}): m / z(\%)=217(12)[\mathrm{M}+1]^{+}, 216(100)\left[\mathrm{M}^{+}\right], 188(44)$ $\left[\mathrm{C}_{11} \mathrm{H}_{12} \mathrm{~N}_{2} \mathrm{O}\right]^{+}, 173$ (82), 159 (30), 130 (10), 117 (14), 89 (9), 77 (9) $\left[\mathrm{C}_{6} \mathrm{H}_{5}\right]^{+}, 63(14)$.

HRMS (EI, $\mathrm{M}^{+}$): $m / z$ calcd for $\mathrm{C}_{12} \mathrm{H}_{12} \mathrm{~N}_{2} \mathrm{O}_{2}$ : 216.0898; found: 216.0898 .

\section{Funding Information}

C.C.M. acknowledges Science and Engineering Research Board (SERB), New Delhi and NIT Manipur for the financial support in the form of research grant (ECR/2016/000337). R.G., N.V., D.K. and A.K.K. are grateful to the Ministry of Human Resource and Development (MHRD), New Delhi for Fellowship support.

\section{Acknowledgment}

We acknowledge Mr. M. Wolf (Institut für Chemie, Universität Hohenheim) and the central instrumental facilities at the Indian Institute of Technology, Guwahati for recording NMR and Mass spectra. We sincerely thank Professors T. Punniyamurthy, V. Satheesh and R. Bag from Department of Chemistry, Indian Institute of Technology, Guwahati for sample analysis and research support.

\section{Supporting Information}

Supporting information for this article is available online at https://doi.org/10.1055/s-0036-1591572.

\section{References}

(1) (a) Royer, J. Asymmetric Synthesis of Nitrogen Heterocycles; Wiley-VCH Verlag: Weinheim, 2009. (b) Huang, Y.; Khoury, K.; Dömling, A. In Synthesis of Heterocycles via Multicomponent Reactions I; Orru, R. V. A.; Ruijter, E., Eds.; Springer: Berlin, Heidelberg, 2010, p 85. (c) Wolfe, J. P.; Neukom, J. D.; Mai, D. H. Synthesis of Saturated Five-Membered Nitrogen Heterocycles via PdCatalyzed C-N Bond-Forming Reactions; Wiley-VCH Verlag: Weinheim, 2011. (d) Eicher, T.; Hauptmann, S.; Speicher, A. Chemistry of Heterocycles: Structure, Reactions, Synthesis, and Applications; Wiley: Somerset, NJ, 2013. (e) Wolfe, J. P. Synthesis of Heterocycles via Metal-Catalyzed Reactions That Generate One or More Carbon-Heteroatom Bonds; Springer: Berlin, Heidelberg, 2013. (f) Sammes, P. G. Chem. Rev. 1976, 76, 113. (g) Fodor, 
L.; Szabó, J.; Bernáth, G.; Sohár, P.; Maclean, D. B.; Smith, R. W.; Ninomiya, I.; Naito, T. J. Heterocycl. Chem. 1989, 26, 333. (h) Diurno, M. V.; Mazzoni, O.; Izzo, A. A.; Bolognese, A. Farmaco 1997, 52, 257. (i) Cecchetti, V.; Filipponi, E.; Fravolini, A.; Tabarrini, O.; Xin, T. Bioorg. Med. Chem. 1997, 5, 1339. (j) Deziel, R.; Malenfant, E. Bioorg. Med. Chem. Lett. 1998, 8, 1437. (k) Armenise, D.; Trapani, G.; Stasi, F.; Morlacchi, F. Arch. Pharm. 1998, 331, 54. (l) Feher, M.; Schmidt, J. M. J. Chem. Inf. Comput. Sci. 2002, 43, 218. (m) Hilton, S. T.; Motherwell, W. B.; Potier, P.; Pradet, C.; Selwood, D. L. Bioorg. Med. Chem. Lett. 2005, 15, 2239. (n) Song, Z.-C.; Ma, G.-Y.; Lv, P. C.; Li, H. Q.; Xiao, Z. P.; Zhu, H. L. Eur. J. Med. Chem. 2009, 44, 3903. (o) Gomez-, Monterrey. I.; Bertamino, A.; Porta, A.; Carotenuto, A.; Musella, S.; Aquino, C.; Granata, I.; Sala, M.; Brancaccio, D.; Picone, D.; Ercole, C.; Stiuso, P.; Campiglia, P.; Grieco, P.; Ianelli, P.; Maresca, B.; Novellino, E. J. Med. Chem. 2010, 53, 8319. (p) Kaur, S. P.; Rao, R.; Nanda, S. Int. J. Pharm. 2010, 402, 37. (q) Leeson, P. D.; St-Gallay, S. A.; Wenlock, M. C. MedChemComm 2011, 2, 91. (r) Kaur, S. P.; Rao, R.; Nanda, S. Int. J. Pharm. Pharm. Sci. 2011, 3, 30. (s) Jarvis, C. L.; Richers, M. T.; Breugst, M.; Houk, K. N.; Seidel, D. Org. Lett. 2014, 16, 3556. (t) Feng, H.; Jia, H.; Sun, Z. Adv. Synth. Catal. 2015, 357, 2447.

(2) (a) Lovering, F.; Bikker, J.; Humblet, C. J. Med. Chem. 2009, 52, 6752. (b) Schnurch, M.; Dastbaravardeh, N.; Ghobrial, M.; Mrozek, B. D.; Mihovilovic, M. Curr. Org. Chem. 2011, 15, 2694. (c) Ritchie, T. J.; Macdonald, S. J. F.; Young, R. J.; Pickett, S. D. Drug Discovery Today 2011, 16, 164. (d) Zhu, Z.; Tang, X.; Li, J.; Li, X.; Wu, W.; Deng, G.; Jiang, H. Org. Lett. 2017, 19, 1370. (e) Song, P.; Yu, P.; Lin, J.-S.; Li, Y.; Yang, N.-Y.; Liu, X.-Y. Org. Lett. 2017, 19, 1330. (f) Yan, F.; Liang, H.; Song, J.; Cui, J.; Liu, Q.; Liu, S.; Wang, P.; Dong, Y.; Liu, H. Org. Lett. 2017, 19, 86.

(3) (a) Colby, D. A.; Bergman, R. G.; Ellman, J. A. Chem. Rev. 2010, 110, 624. (b) Engle, K. M.; Mei, T. S.; Wasa, M.; Yu, J.-Q. Acc. Chem. Res. 2011, 45, 788. (c) Lu, P.; Wang, Y. Chem. Soc. Rev. 2012, 41, 5687. (d) El-salam, N. M. A.; Mostafa, M. S.; Ahmed, G. A.; Alothman, O. Y. J. Chem. 2013, 1. (e) Azab, M. E.; Youssef, M. M.; El-Bordany, E. A. Molecules 2013, 18, 832. (f) Salem, M. S.; Sakr, S. I.; El-Senousy, W. M.; Madkour, H. M. F. Arch. Pharm. (Weinheim) 2013, 346, 766. (g) Xu, X.; Doyle, M. P. Acc. Chem. Res. 2014, 47, 1396. (h) Xie, J.; Pan, C.; Abdukadera, A.; Zhu, C. Chem. Soc. Rev. 2014, 43, 5245. (i) Cao, X.; Sun, Z.; Cao, Y.; Wang, R.; Cai, T.; Chu, W.; Hu, W.; Yang, Y. J. Med. Chem. 2014, 57, 3687. (j) Chen, Y.; Yu, K.; Tan, N. Y.; Qiu, R. H.; Liu, W.; Luo, N. L.; Tong, L.; Au, C. T.; Luo, Z. Q.; Yin, S. F. Eur. J. Med. Chem. 2014, 79, 391. (k) Sharma, U. K.; Sharma, N.; Vachhani, D. D.; der Eycken, E. V. V. Chem. Soc. Rev. 2015, 44, 1836. (l) Yang, L.; Huang, H. Chem. Rev. 2015, 115, 3468. (m) Martins, P.; Jesus, J.; Santos, S.; Raposo, L. R.; Roma-Rodrigues, C.; Baptista, P. V.; Fernandes, A. R. Molecules 2015, 20, 16852. (n) Yu, J.-T.; Pan, C. Chem. Commun. 2016, 2220.

(4) (a) Felpin, F.-X.; Lebreton, J. Eur. J. Org. Chem. 2003, 3693. (b) Ragaini, F.; Cenini, S.; Brignoli, D.; Gasperini, M.; Gallo, E. J. Org. Chem. 2003, 68, 460. (c) Smitrovich, J. H.; Davies, I. W. Org. Lett. 2004, 6, 533. (d) Pyne, S. G.; Davis, A. S.; Gates, N. J.; Hartley, J. P.; Lindsay, K. B.; Machan, T.; Tang, M. Synlett 2004, 2670. (e) Coldham, I.; Hufton, R. Chem. Rev. 2005, 105, 2765. (f) Bellina, F.; Rossi, R. Tetrahedron 2006, 62, 7213. (g) Merisor, E.; Conrad, J.; Klaiber, I.; Mika, S.; Beifuss, U. Angew. Chem. Int. Ed. 2007, 46, 3353. (h) Merisor, E.; Conrad, J.; Mika, S.; Beifuss, U. Synlett 2007, 2033. (i) Merisor, E.; Beifuss, U. Tetrahedron Lett. 2007, 48, 8383. (j) Ferretti, F.; Formenti, D.; Ragaini, F. Rend.
Lincei 2017, 28, 97. (k) El-Atawy, M. A.; Ferretti, F.; Ragaini, F. Eur. J. Org. Chem. 2017, 1902. (l) Formenti, d.; Ferretti, F.; Ragaini, F. ChemCatChem 2018, 10, 148.

(5) Mali, M. Synth. Catal. 2017, 2, 2.

(6) (a) Cadogan, J. I. G.; Cameron-Wood, M. Proc. Chem. Soc. (London) 1962, 361. (b) Cadogan, J. I. G.; Cameron-Wood, M.; Mackie, R. K.; Searle, R. J. G. J. Chem. Soc. 1965, 4831. (c) Sundberg, R. J. Tetrahedron Lett. 1965, 477. (d) Cadogan, J. I. G.; Todd, M. J. J. Chem. Soc. C 1969, 2808. (e) Ho, T. L.; Hsieh, S. Y. Helv. Chim. Acta 2006, 89, 111. (f) Freeman, A. W.; Urvoy, M.; Criswell, M. E. J. Org. Chem. 2005, 70, 5014. (g) Sanz, R.; Escribano, J.; Pedrosa, M. R.; Aguado, R.; Arnaiz, F. J. Adv. Synth. Catal. 2007, 349, 713.

(7) (a) Crotti, C.; Cenini, S.; Rindone, B.; Tollari, S.; Demartin, F. J. Chem. Soc., Chem. Commun. 1986, 784. (b) Annunziata, R.; Cenini, S.; Palmisano, G.; Tollari, S. Synth. Commun. 1996, 26, 495. (c) Söderberg, B. C. G.; Wallace, J. M.; Tamariz, J. Org. Lett. 2002, 4, 1339. (d) Scott, T. L.; Söderberg, B. C. G. Tetrahedron Lett. 2002, 43, 1621. (e) Han, R.; Chen, S.; Lee, S. J.; Qi, F.; Wu, X.; Kim, B. H. Heterocycles 2006, 68, 1675. (f) Wallace, J. M.; Söderberg, B. C. G.; Tamariz, J.; Akhmedov, N. G.; Hurley, M. T. Tetrahedron 2008, 64, 9675.

(8) (a) Fernandes, A. C.; Romáo, C. C. Tetrahedron 2006, 62, 9650. (b) Fernandes, A. C.; Romáo, C. C. Tetrahedron Lett. 2007, 48, 9176. (c) Reis, P. M.; Costa, P. J.; Romáo, C. C.; Fernandes, J. A.; Calhorda, M. J.; Royo, B. Dalton Trans. 2008, 1727. (d) Reis, P. M.; Royo, B. Tetrahedron Lett. 2009, 50, 949.

(9) (a) Alper, H.; Edward, J. T. Can. J. Chem. 1970, 48, 1543. (b) Crotti, C.; Cenini, S.; Bossoli, A.; Rindone, B.; Demartin, F. J. Mol. Catal. 1991, 70, 175. (c) Pizzotti, M.; Cenini, S.; Quici, S.; Tollari, S. J. Chem. Soc., Perkin Trans. 2 1994, 913. (d) For a review, see: Söderberg, B. C. G. Curr. Org. Chem. 2000, 4, 727. (e) Smitrovich, J. H.; Davies, I. W. Org. Lett. 2004, 6, 533. (f) Vodnala, N.; Kaldhi, D.; Polina, S.; Putta, V. P. R. K.; Gupta, R.; Promily, S. C. P.; Linthoinganbi, R. K.; Singh, V.; Malakar, C. C. Tetrahedron Lett. 2016, 57, 5695.

(10) (a) Pizzotti, M.; Cenini, S.; Psaro, R.; Costanzi, S. J. Mol. Catal. 1990, 63, 299. (b) Crotti, C.; Cenini, C. J. Chem. Soc., Faraday Trans. 1991, 87, 2811. (c) Crotti, C.; Cenini, S.; Ragaini, F.; Porta, F.; Tollari, S. J. Mol. Catal. 1992, 72, 283.

(11) Sanz, R.; Escribano, J.; Aguado, R.; Pedrosa, M. R.; Arnáiz, F. J. Synthesis 2004, 1629.

(12) (a) Malakar, C. C.; Merisor, E.; Conrad, J.; Beifuss, U. Synlett 2010, 1766. (b) Moustafa, A. H.; Malakar, C. C.; Aljaar, N.; Merisor, E.; Conrad, J.; Beifuss, U. Synlett 2013, 24, 1573. (c) Siddiqui, I. R.; Srivastava, A.; Singh, A.; Shamim, S.; Rai, P. RSC Adv. 2015, 5, 5256. (d) Vodnala, N.; Kaldhi, D.; Gupta, R.; Polina, S.; Putta, V. P. R. K.; Promily, S. C. P.; Linthoinganbi, R. K.; Singh, V.; Malakar, C. C. ChemistrySelect 2016, 1, 5784.

(13) Garcia, N.; Garcia-Garcia, P.; Fernindez-Rodriguez, M. A.; Rubio, R.; Pedrosa, M. R.; Arnaiz, F. J.; Sanz, R. Adv. Synth. Catal. 2012, 354, 321; and references cited therein.

(14) Gerber, N. N. J. Org. Chem. 1967, 32, 4055.

(15) Toshima, K.; Takano, R.; Ozawa, T.; Matsumura, S. Chem. Commun. 2002, 212.

(16) (a) Anderson, R. K.; Carter, S. D.; Cheeseman, G. W. H. Tetrahedron 1979, 35, 2463. (b) Zhang, Z.; Yin, Z.; Kadow, J. F.; Meanwell, N. A.; Wang, T. Synlett 2004, 2323.

(17) (a) Kidani, Y. Chem. Pharm. Bull. 1959, 7, 88. (b) Kumar, B. S. P. A.; Madhav, B.; Reddy, K. H. V.; Nageswar, Y. V. D. Tetrahedron Lett. 2011, 52, 2862. 